\title{
Does duration of stenting increase the risk of clinical infection?
}

\author{
Tuncay Toprak, Aytaç Şahïn, Musab Ali Kutluhan, Korhan Akgul, Yavuz Onur Danacioglu, \\ Mehmet Akif Ramazanoglu, Ayhan Verit \\ Department of Urology, Fatih Sultan Mehmet Training and Research Hospital, Istanbul, Turkey.
}

\begin{abstract}
Summary Objective: We investigated when an indwelling ureteral catheter should be withdrawn for infection and evaluated the importance of urinary cultures in identifying colonized microorganisms and define the bacterial flora encountered in the study. Moreover, this study tried to determine the clinical role of stent culture in clinical practice.

Material and methods: The study was conducted between June 2018 and February 2019. Patients with ureteral stent implantation after endoscopic ureteral stone treatment were divided into two groups and each group consisted of 45 patients. Ureteral catheter was removed 15 and 30 days after ureteral stone treatment in group 1 and 2, respectively, and transferred for microbiological examination. The urine culture was obtained before and after ureteral stent implantation. The groups were compared in terms of demographics, urine and catheter cultures results. Urine analysis and catheter culture results were also compared.

Results: Demographic data of patients were similar in both groups. 3 patients in group 1 and 12 patients in group 2 had positive urine culture before catheter retraction; 2 of 45 and 6 of 45 patients had positive catheter culture in group 1 and 2 , respectively. Although 2 patients in group 1 and 4 patients in group 2 had urine culture sterile, they had growth in catheter culture. In Group 1, 1 of the microorganisms was E. fecalis and 1 was E. coli. In Group 2, 2 cases were E. fecalis, 3 were E. coli and 1 was MRSE. There was no significant difference between the urine analysis results of the patients before catheter retraction and catheter culture positivity. Conclusions: Pre-operative urine culture does not exclude catheter colonization, and the prolonged duration of the catheter associated with greater colonization and may be associated urinary tract infection. Ureteral catheter should be removed as early as possible.
\end{abstract}

KEY WORDS: Duration; Ureteral stents; Colonization.

Submitted 15 June 2019; Accepted 23 July 2019

\section{INTRODUCTION}

Ureteral stenting is commonly used for drainage of the obstructed or infected upper urinary tract. Although ureteral stent application is not routinely recommended after each ureteroscopy (1) ureteral stents were inserted before the procedure to relieve pain to 7 to $68 \%$ of patients who underwent ureteroscopy (2). Ureteral stent is often colonized and incrustated, because it is in direct contact with urine after insertion (3) and sterile urine cultures do not exclude bacterial colonization on ureter- al stents and postoperative urinary tract infection (4). Many studies indicated there is no significant difference between stents and urine cultures, complicating the selection of appropriate antibiotics even when bacteria are identified in urine culture $(5,6)$. We investigated when an indwelling ureteral catheter should be withdrawn for infection and evaluated the importance of urinary cultures in identifying colonized microorganisms and define the bacterial flora encountered in the study. Moreover, this study tried to determine the clinical role of stent cultures in clinical practice.

\section{MATERIAL AND METHODS}

This prospective study was approved by the institutional ethics committee of Fatih Sultan Mehmet Training and Research Hospital (FSM EAH-KAEK 2019/13) and was conducted between June 2018 and February 2019.

All patients gave an informed consent for participation in the study. Patients who underwent ureteral stent implantation after endoscopic ureteral stone treatment were included in this study. The patients who had positive urine culture before ureteral stone treatment and who underwent ureteroscopy for other reasons and patients who had diabetes mellitus, chronic renal diseases, or immune suppression were not included in this study. Patients were divided into two groups and each group consisted of 45 patients. At the beginning and before catheter retraction urine culture were obtained from mid-stream voided urine. Stents were inserted and removed under aseptic conditions with 22 Fr rigid cystoscope. Intravenous second-generation cephalosporin was given 30-60 minutes before stent placement.

A polyurethane double J stents (DJS; Uromed, Oststeinbek, Germany) was used for insertion. Ureteral catheters were removed 15 and 30 days after ureteral stone treatment in group 1 and 2 , respectively. The ureteral stents were transferred to the microbiological examination immediately. Post-operative antibiotics were not given. Urine culture and ureteral catheter culture results of patients were compared between groups. Urine analysis results and catheter culture results were also compared.

\section{Statistical analysis}

When evaluating the findings obtained in this study, IBM SPSS Statistics 22 for statistical analysis (SPSS IBM, Turkey) 
programs were used. The conformity of the parameters to the normal distribution was evaluated by Shapiro Wilks test. For evaluation of study data, Chi-Square test was used to compare qualitative data as well as descriptive statistical methods. Significance was evaluated as $\mathrm{p}<0.05$.

\section{RESULTS}

A total of 90 patients were included in this study. Patients were randomized into two groups. Patients' characteristics are summarized in Table 1. Male/female ratio was 1.5 in group 1 and 1.25 in group 2 . The mean age was 45.6 in group 1 and 42.7 in group 2. No significant difference was observed between the groups in terms of age and gender. The urine culture of all patients was sterile before catheter insertion. Urine culture taken before catheter retraction was positive in 3 patients in group 1 and 12 patients in group 2. Table 2 shows comparison of bacterial growth between groups.

Patients with positive urine culture were treated with appropriate antibiotics before ureteral catheter with-

Table 1.

Comparison of demographic characteristics between groups.

\begin{tabular}{|lccc|}
\hline & Group $\mathbf{1}(\mathbf{n}=\mathbf{4 5})$ & Group $\mathbf{2}(\mathbf{n}=\mathbf{4 5})$ & P value \\
\hline $\mathrm{M} / \mathrm{F}(\%)$ & $60 / 40$ & $55.5 / 44.5$ & $>0.05$ \\
\hline Age, years, mean & $45.6(\min 19, \max 73)$ & $42.7(\min 23, \max 76)$ & $>0.05$ \\
\hline
\end{tabular}

Table 2.

Comparison of bacterial growth between groups.

\begin{tabular}{|c|c|c|c|}
\hline & Group $1(n=45)$ & Group $2(n=45)$ & $P$ value \\
\hline $\begin{array}{l}\text { Urine culture (at the } \\
\text { beginning or before ureteral } \\
\text { catheter placement) }\end{array}$ & All of them sterile & All of them sterile & \\
\hline $\begin{array}{l}\text { Urine culture (after ureteral } \\
\text { catheter placement or before } \\
\text { ureteral catheter retraction) }\end{array}$ & $\begin{array}{c}3 \text { positive }(6.6 \%) \\
12 \text { positive }(26.6 \%)\end{array}$ & $\begin{array}{l}\text { (2 of them women) } \\
\text { (8 of them women) }\end{array}$ & 0.01 \\
\hline $\begin{array}{l}\text { Catheter culture results } \\
\text { of patients with positive urine } \\
\text { culture before catheter retraction } \\
\text { (after antibiotic treatment) }\end{array}$ & 3 of them sterile & 10 of them sterile & \\
\hline Catheter culture in total & $\begin{array}{l}2 \text { positive (4.4\%) } \\
\text { (1 of them women) }\end{array}$ & $\begin{array}{l}6 \text { positive (13.3\%) } \\
\text { (4 of them women) }\end{array}$ & 0.14 \\
\hline
\end{tabular}

Table 3.

Bacteriology of the cultured ureteral stents.

\begin{tabular}{|lcc|}
\hline & Group 1 $\mathbf{n}(\%)$ & Group 2 $\mathbf{n}(\%)$ \\
\hline Enterococcus fecalis & $1(\% 2.2)$ & $2(\% 4.4)$ \\
\hline MRSE & $0(\% 0)$ & $1(\% 2.2)$ \\
\hline E. coli & $1(\% 2.2)$ & $3(\% 6.6)$ \\
\hline Sterile & $43(\% 95.5)$ & $39(\% 86.6)$ \\
\hline Total & $45(\% 100)$ & $45(\% 100)$ \\
\hline
\end{tabular}

drawal. Three patients with positive urine culture in Group 1 had no bacterial growth in catheter culture after antibiotic treatment. Two of 12 patients with positive urine culture in Group 2 had the same microorganisminduced growth in catheter culture after antibiotic treatment. Although 2 patients in group 1 and 4 patients in group 2 had urine culture sterile, they had growth in catheter culture.

As shown in Table 3; one of the microorganisms isolated from urine culture in Group 1 was E. fecalis and one of was E. coli. In Group 2, 2 cases were E. fecalis, 3 were E. coli and 1 was MRSE. The urine analysis of the patients before the procedure was investigated for nitrite positivity, leukocyte esterase positivity and pyuria and compared with catheter culture results. As shown in Table 4 no statistically significant difference was found between catheter culture and urine analysis results. The duration of surgical procedures ranged from 9 to 37 minutes, but the relationship between the duration of surgery and colonization was not investigated.

\section{Discussion}

Ureteral stents are usually effective and safe in order to deliver urine from kidney to the bladder. However, they can lead to various complications, one of them being urinary infection (7). After stent insertion biofilm formation starts immediately, however, the time required for bacteria to colonize the stent has not yet been defined (3). Several studies showed the ability of uropathogens such as E. coli, Proteus mirabilis, Staphylococcus epidermidis, and Enterococcus faecalis to form biofilms on ureteral stents within 24 hours $(8,9)$. Biofilm formation process on a ureteral catheter is well defined by some studies (10), and begins with the early development of the first membrane on the catheter. Bacteria on this membrane can more easily adhere and multiply. This environment protects bacteria from antibacterial factors (3) and bacteria appear to be more resistant to antibiotics by developing resistance genes to antibiotics (11). Consequently, it is not surprising that stent colonization is frequently encountered. In our study approximately $9 \%$ of our patients hosted one microorganism and $87.5 \%$ of these colonies included Gram-negative bacteria. This rate is similar to rates described by other publications that are below $50 \%$ for a mean catheterization time between 2 and 9 weeks $(6,12-14)$. Stent retention time in the ureter increases the likelihood of biofilm formation and so the duration of stenting is considered to be a critical factor for bacterial proliferation (13). However, some reports $(6,15)$ didn't find a meaningful relationship between positive cultures and catheterization time. In our study, patients in group 2 had more bacterial growth in ureteral stent cultures than group 1 patients. Female gender in

\begin{tabular}{|lcccccc|}
\hline & Group 1 & Group 1 & P value & Group 2 & Group 2 & P value \\
\hline & $\begin{array}{c}\text { Positive catheter } \\
\text { culture }(\mathbf{n}=\mathbf{2})\end{array}$ & $\begin{array}{c}\text { Negative catheter } \\
\text { culture }(\mathbf{n}=\mathbf{4 3})\end{array}$ & $\begin{array}{c}\text { Positive catheter } \\
\text { culture }(\mathbf{n}=\mathbf{6})\end{array}$ & $\begin{array}{c}\text { Negative catheter } \\
\text { culture }(\mathbf{n}=\mathbf{3 9})\end{array}$ & \\
\hline Pyuria $(>5$ leukocytes) & $1(\% 50)$ & $18(\% 41.8)$ & 0.82 & $3(\% 50)$ & $21(\% 53.84)$ & 0.86 \\
\hline Leukocyte esterase positivity & $0(\% 0)$ & $4(\% 9.3)$ & 0.65 & $1(\% 16.6)$ & $3(\% 7.6)$ & 0.47 \\
\hline Nitrite positivity & $0(\% 0)$ & $3(\% 6.9)$ & 0.69 & $2(\% 33.3)$ & $5(\% 12.8)$ & 0.19 \\
\hline
\end{tabular}

Table 4

Comparison of catheter culture and urine analysis. 
various studies was found to be associated with a high rate of sepsis as a result of the high infection rate in this population (16). As expected, in our study $62.5 \%$ of patients with positive ureteral catheter culture were women, but we have not encountered symptomatic infection or sepsis. The relationship between urine and ureteral catheter cultures is not well defined. Lojanapiwat (17) published urine culture results showing colonization in approximately two-thirds of patients, whereas Klis et al. (5) indicated a large inconsistency between urine and ureteral catheter cultures. Our data supports the discordance between preoperative urine and intraoperative stent culture. In this study, 6 patients had positive stent culture despite sterile urine culture. Sterile urine culture in the presence of foreign bodies doesn't prevent stent colonization, and this may cause urinary tract infection (18). Although some studies have reported the opposite (15), in our study, the most common pathogen in ureteral catheter cultures were E. coli and Enterococci. In literature, there are also other publications reporting that E. coli $(4,17)$ and Enterococci $(6)$ are most common in ureteral catheter culture. Kehinde et al. (19) showed that bacteriuria and ureteral stent colonization increased significantly with a longer stenting time, female gender and presence of systemic diseases such as diabetic nephropathy, chronic renal failure and diabetes mellitus and recommended that patient of these categories should have shorter stenting time and antimicrobial prophylaxis to minimize infectious complications. Another study (20) emphasized that early removal of the ureteral stent, 2 weeks after renal transplantation, reduced the rate of urinary tract infection. Although not statistically significant our study gave similar results: longer duration of stenting was associated to higher colonization rate $(4.4 \%$ for stents left for 15 days versus $13.3 \%$ for those left for 30 days). None of our patients had any systemic disease therefore the study of the correlation between presence of pathologies and colonization was not made.

In conclusion, our study shows that results of urine cultures do not represent the results of ureteral stent cultures. E. coli is usually isolated and should be coated with preoperative antibiotics. Our study demonstrates that the stents are colonized under natural conditions and that more awareness should be necessary before using these stents. Our findings also showed that colonization of ureteral stents was not associated with the development of symptomatic infection. We didn't found any symptomatic infection after stent removal and we found a colonization rate of $4.4 \%$ within 15 days and $13.3 \%$ within 30 days.

\section{Limitations of our study}

We have given preoperative antibiotic treatment which may have affected bacterial flora. Although a study (5) showed that colonization throughout the stent is consistent, we didn't investigate different ureteral stent segments which could be colonized by different pathogens. Our bacterial profile depends from local flora and could be not transferable to other centers. Finally, stone culture was not done although bacteria within the stone could affect ureteral colonization.

\section{Conclusions}

The clinical significance of bacterial colonization of ureteral stent seems to be low, and it seems that ureteral stents are safer, especially within 15 days when colonization is very low. Urine analysis and urine culture results are not related with ureteral stent culture and prolongation of ureteral stent increases colonization. Further studies are needed to determine the optimal indwelling time of ureteral stent after endoscopic ureter stone treatment. Knowing the bacteriological flora of an institution is useful for evidence-based prophylactic and therapeutic application. It is not recommended to routinely send the stents to microbiological examination because it is not cost effective and increases the workload to the microbiology laboratory. Stents should be withdrawn immediately if no more required.

\section{Informed consent}

Ureteral stent is frequently inserted after ureteral stone treatment. Our study named 'DOES STENT DURATION INCREASE THE RISK OF CLINICAL INFECTION?’ will investigate the relationship between the duration of these ureteral stents with infection. The ureteral stent of some patients will be taken 15 days after the stone treatment and some of them will be taken 30 days later and sent to the microbiological examination. Our research is multicentered and will be between September 2018 and January 2019. A total of 100 patients were planned to be included in the study. Patients will be randomized into two groups. In the event of any unintended or unexpected health problems directly or indirectly related to the research, any medical intervention will be provided by us without any charge. You are completely free to participate in the research. Failure to participate in this study will not necessarily affect your current treatment or relationship with your physician. You have the right to withdraw from the work by giving notice at any time; and if deemed necessary, you may be excluded from research by the investigator, provided that your medical condition is not harmed. If you participate in the research, you will not be charged any fees or charges for any expenses incurred in the study. The sample taken from you for research will be used only for this study. In addition, your information at the end of the research will serve only scientific purposes without your identity being disclosed.

\section{Author's contribution}

Toprak: Project development, Data Collection, Manuscript writing; Sahin: Data Collection, Statistical analysis; Kutluhan: Manuscript writing; Akgul: Revision; Danacioglu: Data Collection; Ramazanoglu: Data Collection; Verit: Revision.

\section{REFERENCES}

1. Blackmur JP, Maitra NU, Marri RR, et al., Analysis of factors' association with risk of postoperative urosepsis in patients undergoing ureteroscopy for treatment of stone disease. J Endourol. 2016; 30: 963-969. 
2. Sivalingam S, Stormont IM, Nakada SY. Contemporary practice patterns in the management of acute obstructing ureteral stones. J Endourol. 2015; 29: 736-740.

3. Zumstein V, Betschart P, Albrich WC, et al. Biofilm formation on ureteral stents-Incidence, clinical impact, and prevention. Swiss Med Wkly. 2017; 147:w14408.

4. Farsi HM, Mosli HA, Al-Zemaity MF, et al. Bacteriuria and colonization of double-pigtail ureteral stents: long-term experience with 237 patients. J Endourol. 1995; 9:469-72.

5. Klis R, Korczak-Kozakiewicz E, Denys A, et al. Relationship between urinary tract infection and self-retaining Double-J catheter colonization. J Endourol. 2009; 23:1015-1019.

6. Lifshitz DA, Winkler HZ, Gross M, et al. Predictive value of urinary cultures in assessment of microbial colonization of ureteral stents. J Endourol. 1999; 13:735-8.

7. Vallejo JH, Burgos FR, Alvarez JA, et al. Double J ureteral catheter. Clinical complications. Arch Esp Urol. 1998; 51:361-373.

8. Stickler DJ. Bacterial biofilms in patients with indwelling urinary catheters. Nature Reviews Urology. 2008; 5:598.

9. Gabi M, Hefermehl L, Lukic D, et al. Electrical microcurrent to prevent conditioning film and bacterial adhesion to urological stents. Urol Res, 2011; 39:81-88.

10. Reid G, Denstedt JD, Kang YS, et al. Microbial adhesion and biofilm formation on ureteral stents in vitro and in vivo. J Urol. 1992; 148:1592-1594

11. Kiran MD, Giacometti A, Cirioni O, Balaban N. Suppression of biofilm related, device-associated infections by staphylococcal quorum sensing inhibitors. Int J Artif Organs. 2008; 31:761-70.
12. Paick SH, Park HK, Oh SJ, Kim HH. Characteristics of bacterial colonization and urinary tract infection after indwelling of double-J ureteral stent. Urology. 2003; 62:214-217.

13. Kehinde EO, Rotimi VO, Al-Hunayan A, et al. Bacteriology of urinary tract infection associated with indwelling J ureteral stents. J Endourol. 2004; 18:891-896.

14. Aydin HR, Irkilata L, Aydin M, et al. Incidence of bacterial colonisation after indwelling of double-J ureteral stent. Arch Ital Urol Androl. 2016; 87:291-4.

15. Kozyrakis D, Perikleous S, Chatzistamou S-E, et al. Is there a role for double J stent culture in contemporary urology? Urol Int. 2018; 100:203-208.

16. De La Rosette J, Denstedt J, Geavlete P, et al., The clinical research office of the endourological society ureteroscopy global study: indications, complications, and outcomes in 11,885 patients. J Endourol. 2014; 28:131-139.

17. Lojanapiwat B. Colonization of internal ureteral stent and bacteriuria. World J Urol. 2006; 24:681-683.

18. Grabe M, Botto H, Cek M, et al. Preoperative assessment of the patient and risk factors for infectious complications and tentative classification of surgical field contamination of urological procedures. World J Urol. 2012; 30:39-50.

19. Kehinde EO, Rotimi VO, Al-Awadi KA, et al. Factors predisposing to urinary tract infection after J ureteral stent insertion. J Urol. 2002; 167:1334-7.

20. Coskun AK, Harlak A, Ozer T, et al. Is removal of the stent at the end of 2 weeks helpful to reduce infectious or urologic complications after renal transplantation? Transplant Proc. 2011; 43:813-5

\author{
Correspondence \\ Tuncay Toprak, MD (Corresponding Author) \\ drtuncay55@hotmail.com \\ AytaçSahïn, MD \\ draytacsahin@gmail.com \\ Musab Ali Kutluhan, MD \\ dr.musab151@gmail.com \\ Korhan Akgul, MD \\ korhanakgul@gmail.com \\ Ayhan Verit, MD \\ veritayhan@yahoo.com \\ Department of Urology, Fatih Sultan Mehmet Training and Research Hospital, \\ Istanbul (Turkey) \\ Yavuz Onur Danacioglu, MD \\ dr_yonur@hotmail.com \\ Department of Urology, Bakirkoy Dr. Sadi Konuk Training and Research \\ Hospital, Istanbul (Turkey) \\ Mehmet Akif Ramazanoglu, MD \\ maramazanoglu@hotmail.com \\ Urology, Rize State Hospital, Rize (Turkey)

